\title{
Developing haptic caries simulation for dental education
}

\section{Cecilie Osnes* and Andrew Keeling}

School of Dentistry, University of Leeds, Leeds, LS2 9LU, UK

${ }^{*}$ Corresponding author at: Room 6.008, Worsley Building, Clarendon Way, Leeds, LS2 9LU, UK. Email: C.A.Osnes@leeds.ac.uk

Date accepted for publication: 19 June 2017

\section{Abstract}

Background: Treating tooth decay (caries), an essential skill for dental students to learn, relies largely on tactile investigation. Most dental schools use plastic or extracted teeth to teach caries removal and cavity design. However, plastic teeth cannot replicate the 'feel' of caries, limiting the available practice exercises to manual dexterity rather than cavity design guided by pathology. Virtual haptic simulation is emerging as a viable adjunct to traditional methods, with potential to simulate dental tissues and pathology. Aim: To develop virtual haptic caries simulations, enabling students to practice the tactile and cognitive aspects of caries removal and cavity design in a controlled environment on the Moog Simodont Dental Trainer. Methods: Custom software was written to enable the creation of blocks containing unique variations of caries. Each block was produced with segment data to enable automatic marking of students' attempts. This caries block generation process enabled the creation of a large library of disparate carious models calling for a unique solution for each case. Results: A library of caries blocks was built. The blocks hold varying shape, depth and amount of caries within a simulated tooth consisting of enamel, dentine and pulp tissue. The design of the block encouraged the user to follow correct clinical protocols to treat the lesion, while preserving the maximum amount of healthy tissue. Conclusions: We present a novel haptic caries simulation encouraging correct clinical technique based on tactile sensation and realistic disease patterns. The exercises provide a safe environment for novice dental students to practice caries removal.

Keywords: dentistry; education; haptic simulation; virtual; cariology; caries removal

\section{Introduction}

Introducing undergraduate dental students to the operative treatment of dental caries (tooth decay) during preclinical teaching is a challenge. Cavity preparation is dictated by the carious lesion; the process of removing caries requires the dentist to determine the pathological state of tissue within a tooth. This is achieved through tactile probing and, to a lesser extent, visual inspection. Carious lesions generally spread along the junction between enamel and dentine (the amelodentinal junction $[\mathrm{ADJ}])$, then advance deeper into the dentine. This leads to undermined enamel and a carious ADJ, which will initially guide the clinician in preparing the cavity outline. Once a sound cavity outline is achieved, deeper dentinal caries can be partially removed (Fig. 1a-g).

This 'cognitive' process of designing the cavity outline before using less aggressive burs and hand excavators on the deeper carious tissue is poorly simulated using current techniques, despite being arguably the most important skill required for successful cavity design. For example, in UK dental schools, cavity preparation is predominantly taught using plastic teeth. This is little more than a manual dexterity exercise, and may even encourage bad habits such as the extended use of high-speed diamond burs in deeper regions of the tooth (Fig. 1h). Attempts to manufacture caries into plastic teeth have been shown to create inconsistent results and may even disadvantage some students. ${ }^{1}$ The use of carious extracted teeth is diminishing because of difficulties obtaining sufficient teeth of adequate quality, and the inconsistent learning experience they provide to individual students. This inconsistency has been shown to extend to the individual feedback given by different tutors ${ }^{2}$ during the teaching of caries removal. In addition, little correlation has been found between performance on preclinical caries tests and performance on more realistic carious teeth, ${ }^{3}$ or indeed clinical performance in general. ${ }^{4}$

The use of haptic simulators in dental education allows students to practice hand/eye co-ordination, manual dexterity and mirror handling with minimal staff time and 

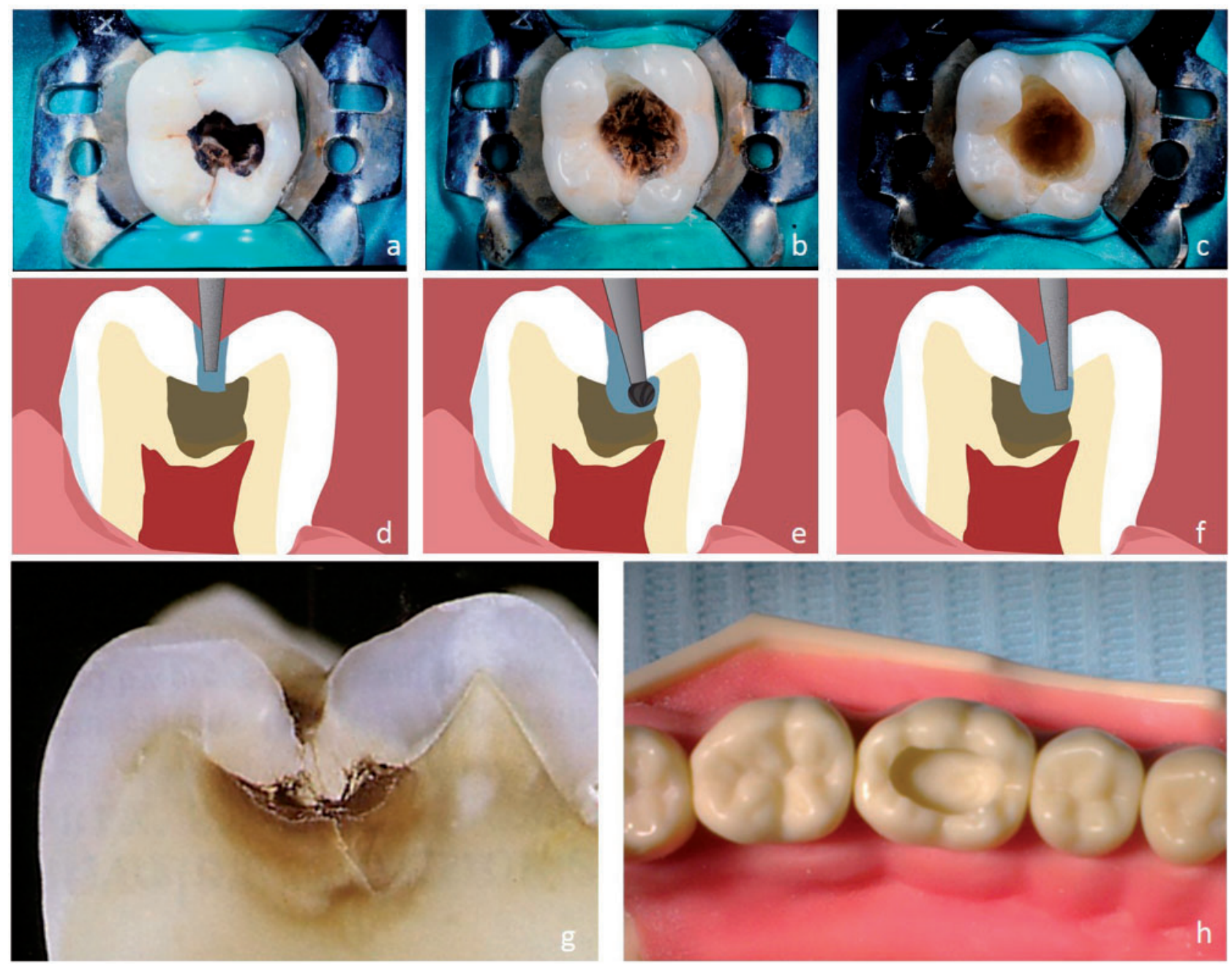

Figure 1. The cognitive process of caries removal. The untreated lesion (a) presents with hidden caries spreading along the ADJ, undermining the enamel as shown in cross-section (g). The first task is to access the cavity and clear the caries from the ADJ using rotary high-speed diamond burs to cut through the hard enamel (d) and stainless steel slow-speed rose-head burs to remove softer dentinal caries just under the ADJ (e). This process of removing soft carious dentine (slow speed) then hard, undermined enamel (high speed) is repeated around the periphery of the lesion until the entire margin of the cavity is caries free (b and f). Note that the outline form of the cavity is designed on the basis of the clinical feel of the tooth tissue, and cannot be precisely predicted in advance. Finally, deeper caries is excavated using gentle rotary instruments and hand excavators (c). Deep dentinal caries may be left in situ and should heal once an adequate restoration is placed. Contrast the clinical protocol (a-g) to the commonly taught preclinical method of drilling a prescribed sized cavity in a plastic tooth (h). The latter does not encourage the correct use of instruments or engender a cognitive approach to cavity design.

resources, ${ }^{5,6}$ and in a safe environment. Evidence suggests that there is a link between simulator ability and operative skills, indicating some benefit to using haptic simulation in undergraduate dental teaching. ${ }^{7-9}$ Furthermore, simulators can enable entire cohorts to attempt identical exercises, making dental simulators ideal for examinations and assessments, ensuring a level of consistency and validity that may be challenging to replicate in a traditional skills lab., ${ }^{2,4}$ Simulation exercises can also offer instantaneous, automated marking, enabling objective, performance-based assessment of student's abilities.

Haptic simulation might be well suited to complement traditional caries teaching, by providing a uniform set of exercises designed to simulate the cognitive process of carious cavity design. Several attempts have been made to simulate carious cavities using haptics and to assess their construct validity. For example, Eve et al. ${ }^{8}$ created a uniformly softer shape within a harder haptic tooth. The carious shape was coloured green, and the performance of experienced clinicians and novices was assessed. Unfortunately, the nature of the lesion was not particularly realistic, as there was no undermined enamel or spread of caries along the ADJ. The exercise was closer to a 'manual dexterity' task of neatly removing the soft, green material, rather than a cognitive cavity design exercise. Other groups have simulated occlusal caries with a more realistic, smaller 
surface cavitation leading to wider caries inside the tooth. ${ }^{10}$ However, the lesion was coloured black, adding a clear visual guide (something that is not always present clinically). In addition, the scoring system only considered the volume of caries removed and not the degree of healthy tooth damaged in the process. Furthermore, the system was not displayed in three-dimensions (3D), a feature that has been shown to enhance dental clinical simulation. ${ }^{11}$

Because of the anisotropic nature of carious cavities, computed tomographic (CT) scans of carious teeth do not produce a realistic representation of caries in haptic simulators. In addition, the relatively intact enamel layer, which often covers a carious lesion, is poorly preserved in CT scans. Hence, an alternative method for recreating the idiosyncratic tactile gradient and range within carious lesions, along with the partially intact enamel covering, is required.
We present a novel method for creating tactile carious pathology using a haptic dental trainer (Simodont, MOOG, Nieuw-Vennep). Our method enables the creation of natural lesions within 'blocks' of virtual enamel, dentine and pulp. The exercises aim to encourage the cognitive aspects of carious cavity design as well as improve the realism of simulated pathology. A scoring system for instant feedback, or for objective assessment, is provided, which takes into account the percentage of healthy tooth damaged and the location within the cavity of the caries that is removed.

\section{Materials and method}

Virtual blocks of varying haptic densities were built using custom software. These caries blocks were cubes of uniform scale consisting of healthy dentine, encased in enamel with a

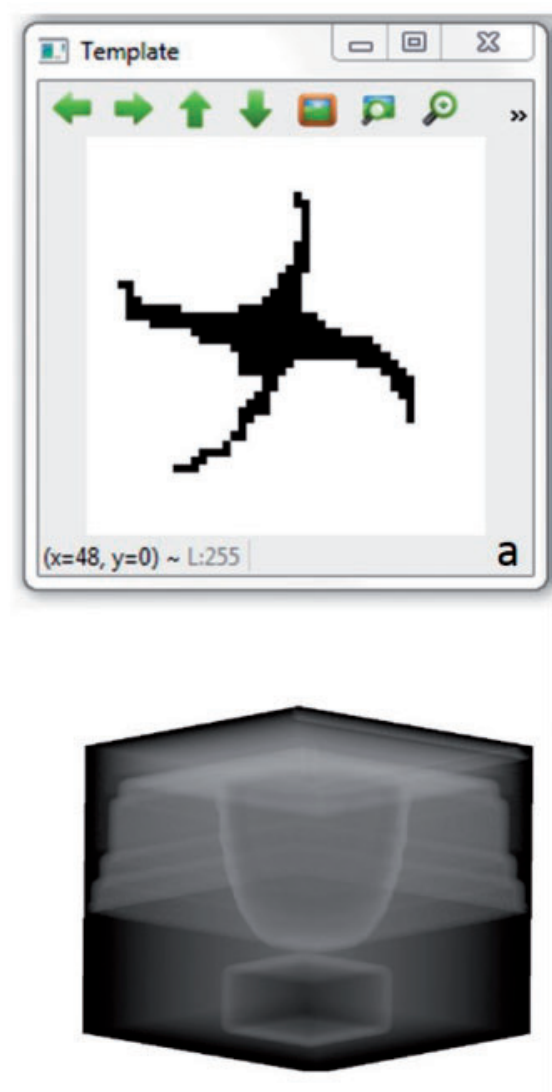

d
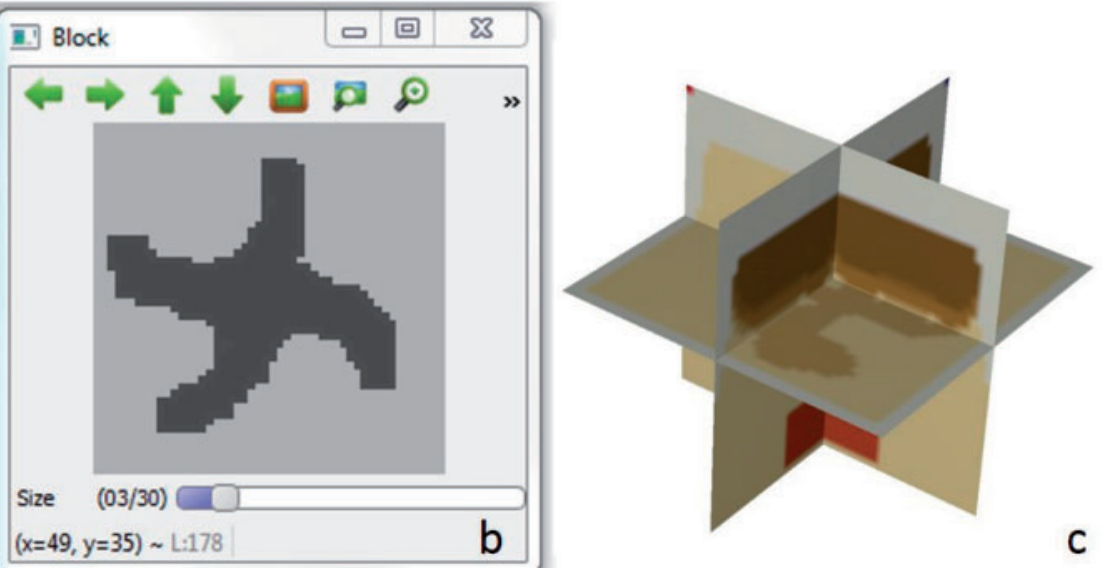

C
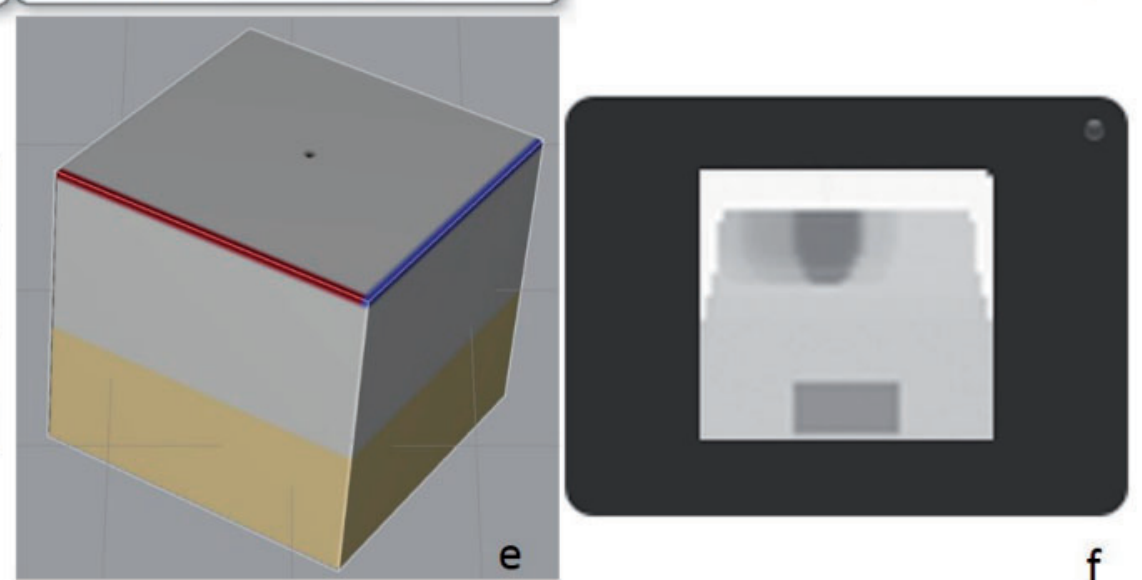

Figure 2. Designing a caries block. A seed image is created that will dictate the shape of the lesion (a). Custom software allows the user to control the extent to which the lesion grows using a slide bar (b). The lesion is then implanted within a block of virtual enamel, dentine and pulp $(c, d)$. The finished model has a very small amount of cavitation on the surface (the black dot visible in (e)), which indicates where the clinician should start cavity preparation. However, the precise shape of the caries is not obvious, and must be discovered using an appropriate clinical technique. The exercise is imported into the Simodont (e) along with an auto-generated radiograph (f). This radiograph would usually be available clinically, and helps to guide the dentist, albeit under the limitation that it is a plain-film 2D image of a 3D lesion. The red and blue lines (e) help the user to orientate the block relative to the radiograph. 
visible access point and an internal pool of carious material of gradual softness.

The realistic densities of healthy enamel and dentine, and the variable density profile of caries, were agreed upon by three experienced dentists using the Simodont. Custom software was written to generate image stacks using the agreed density values. An algorithm was designed for simulating pathologically realistic caries growth, infiltrating along the $\mathrm{ADJ}$, and penetrating deeper into the tooth tissue. A seed image was provided to this algorithm to create a customized carious lesion. The amount, depth, and shape of the caries within the block could be manipulated by altering the seed image and a slider control. The software assigned a density value $(0-255)$ to each voxel in the stack, based on the output of the algorithm. These stacks were later converted into Simodont-compatible volume data. This semi-automatic generation process allowed for a wide range of cases to be built relatively effortlessly (Fig. 2).

In some cases, the carious dentine was visualized as a discoloured region (darker brown), while in others all dentine

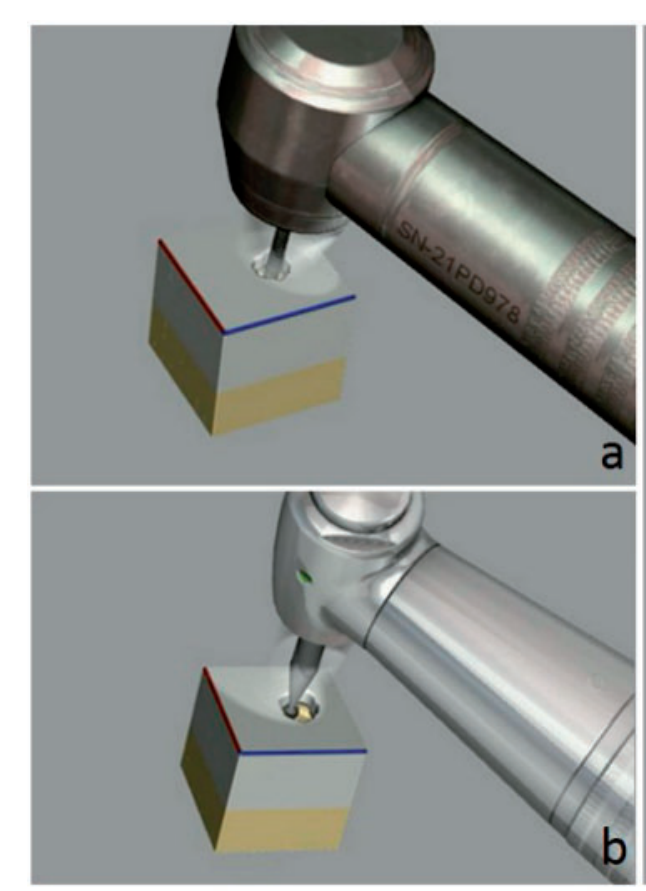

Real time statistics

\section{[Pulp tissue]}

[Dentine]

[Caries]

[Enamel]

\section{[AD] Caries]}

$\mathbf{x}$

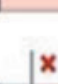

L

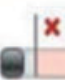

$0 \%$

$0.2 \%$

$0.6 \%$

$3.6 \%$

$56.8 \%$

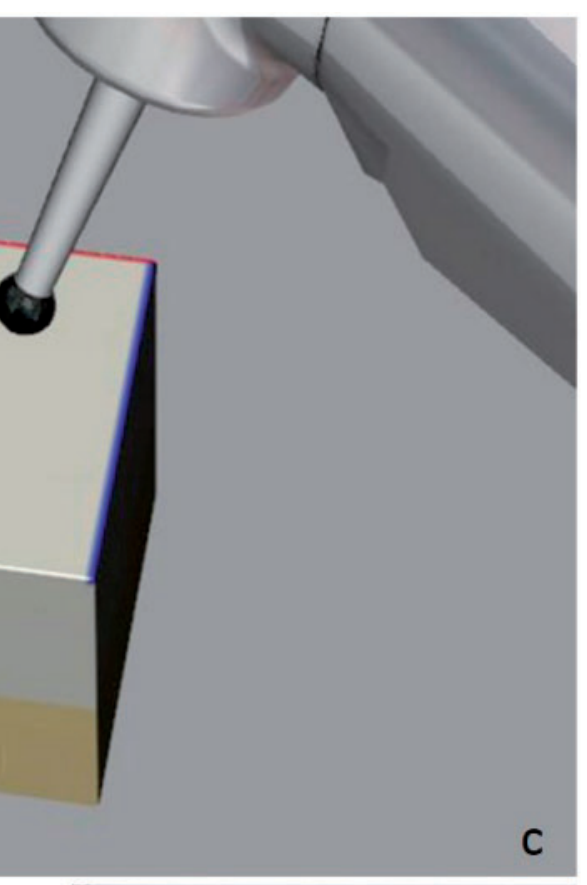

C

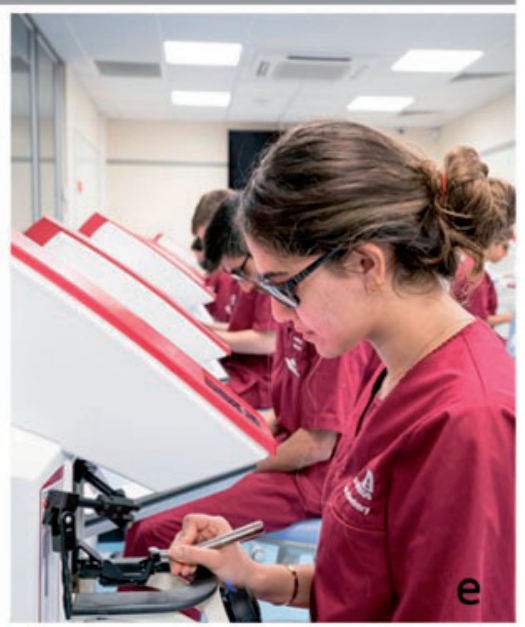

Figure 3. The caries block exercises in use. Access through the enamel is gained using a haptic-rendered high-speed diamond bur (a). The spread of the dentinal caries at the ADJ is then explored using the feel of the slow-speed stainless steel bur (b). Undermined enamel at the cavity margins is removed with the high-speed bur, and this process is repeated until the periphery of the cavity is free of disease. This procedure is underway in (c). Only after the cavity outline has been fully designed is the deeper caries addressed. Some may be left in situ, particularly if its removal is likely to lead to exposure of the pulp tissue. In this example, there is no difference in the colour of healthy and carious dentine, as is sometimes the case clinically; in other caries blocks the carious dentine is discoloured, as can be seen in (Fig. 2c). The tutor can choose whether to allow the student to see real-time feedback during the exercise (d) or not. 
was rendered the same colour whether healthy or carious. This is in line with the clinical presentation, and allowed users to practice on discoloured blocks first, then hone their skills on the more difficult non-discoloured blocks.

To allow for automated marking, segment volumes were generated for each individual case. This enabled the simulator software to track the amount of each material drilled. A distinction was made between caries near the ADJ (which should always be removed), and deeper caries (which may be left in situ) to enable clinically relevant scoring and feedback. This segment data enabled instant on-screen progress tracking, if activated by the tutor, and instant, objective marking of each attempt.

\section{Results}

The process of creating a caries block required approximately 6 minutes of user interaction. Ten such blocks, of varying severity of disease, were uploaded to the simulators and made available as a preclinical undergraduate course.

Informal feedback from students and tutors was positive. An experienced dental tutor commented:

The unique shape of the simulated carious lesion, together with the clinically realistic relative densities of caries, dentine and enamel, challenge the user to adhere to the basic principles of caries removal in order to successfully complete the exercise.

The exercises encouraged the students to approach each cavity preparation cognitively, using the correct instruments for each phase of preparation (Fig. 3).

\section{Discussion}

We have presented a method for creating realistic simulations of carious pathology with objective feedback. The automated scoring required $95 \%$ caries removal from the ADJ segment (as would be expected clinically). Deeper dentinal caries (near the pulp) could be left in situ without penalty to the overall score but unnecessary removal of healthy tooth tissue was penalized. We believe this clinically led automatic feedback is unique to these haptic caries blocks and encourages correct clinical procedure. This is in contrast to the typical experience of training on plastic teeth, which may encourage bad habits such as the inappropriate use of the aggressive high-speed diamond bur in deeper dental tissue.

Because of the limitations of current haptics, the Simodont could not correctly simulate the dental probe, which should generally stick in carious dentine, a key indicator for a clinician. It was decided to limit the instrument selection within the cases to the more realistically rendered high- and slow-speed burs, the latter providing tactile feedback for appropriate caries removal.

Future work is underway to assess the construct validity of the exercises and extend them to mapping the caries algorithm into healthy virtual teeth.

\section{Conclusion}

We have presented the first virtual simulation exercise, to our knowledge, which encourages a cognitive approach to cavity design through correct use of instruments and realistic carious pathology. Haptic simulation enables students to practice procedures currently not available in a traditional preclinical environment. This work may contribute to students' understanding of, and approach to, cavity preparation before clinical exposure. With a library of carious scenarios, we can offer a consistent learning experience to a cohort, yet still offer pathological variety across the cases. The simulations required a cognitive approach to carious cavity design, in contrast to traditional manual dexterity exercises.

\section{Conflict of interest}

The authors have no conflicts of interest.

\section{References}

1. Delgado AJ, Walter R, Behar-Horenstein LS, Boushell LW. Are all dentiform teeth with simulated caries the same? A six-year retrospective study in preclinical operative dentistry. J Dent Educ 2015; 79: 1330-1338.

2. Nascimento MM, Behar-Horenstein LS, Feng X, GuzmánArmstrong S, Fontana M. Exploring how U.S. dental schools teach removal of carious tissues during cavity preparations. J Dent Educ 2017; 81: 5-13.

3. Zietsman C, Engebretson S, Urbankova A. Preclinical operative dentistry performance and association with introduction of caries (abstract). International Association for Dental Research, General Session, 2011. https://iadr.confex. com/iadr/2011sandiego/webprogram/Paper150512.html (accessed 19 June 2017).

4. Fugill M. Defining the purpose of phantom head. Eur J Dent Educ 2013; 17: e1-4.

5. Al-Saud LM, Mushtaq F, Allsop MJ, Culmer PC, Mirghani I, Yates E, et al. Feedback and motor skill acquisition using a haptic dental simulator. Eur J Dent Educ 2016. https://dx.doi. org/10.1111/eje.12214.

6. Bakker D, Lagerweij M, Wesselink P, Vervoorn M. Transfer of manual dexterity skills acquired in the Simodont, a dental 
haptic trainer with a virtual environment, to reality: a pilot study. Bio-Algorithms and MedSystems 2010; 6: 21-24.

7. Mirghani I, Mushtaq F, Allsop MJ, Al-Saud LM, Tickhill N, Potter C, et al. Capturing differences in dental training using a virtual reality simulator. Eur J Dent Educ 2016. http://dx.doi. org/10.1111/eje.12245.

8. Eve EJ, Koo S, Alshihri AA, Cormier J, Kozhenikov M, Donoff RB, et al. Performance of dental students versus prosthodontics residents on a $3 \mathrm{D}$ immersive haptic simulator. J Dent Educ 2014; 78: 630-637.

9. Suebnukarn S, Phatthanasathiankul N, Sombatweroje S, Rhienmora P, Haddawy P. Process and outcome measures of expert/novice performance on a haptic virtual reality system. J Dent 2009; 37: 658-665. https://dx.doi.org/10. 1016/j.jdent.2009.04.008.

10. Yamaguchi S, Yoshida Y, Noborio H, Murakami S, Imazato S. The usefulness of a haptic virtual reality simulator with repetitive training to teach caries removal and periodontal pocket probing skills. Dent Mater J 2013; 32: 847-852. https://doi. org/10.4012/dmj.2013-174.

11. de Boer IR, Wesselink PR, Vervoorn JM. The creation of virtual teeth with and without tooth pathology for a virtual learning environment in dental education. Eur J Dent Educ 2013; 17: 191-197. https://dx.doi.org/10.1111/eje.12027. 